\title{
Stability of casein micelles in milk
}

\author{
R. Tuinier and C. G. de Kruifa) \\ NIZO Food Research, P.O. Box 20, 6710 BA Ede, The Netherlands, and Van't Hoff Laboratory for Physical \\ and Colloid Chemistry, Debye Research Institute, University of Utrecht, Padualaan 8, 3584 CH Utrecht, \\ The Netherlands
}

(Received 20 October 2001; accepted 18 April 2002)

\begin{abstract}
Casein micelles in milk are proteinaceous colloidal particles and are essential for the production of flocculated and gelled products such as yogurt, cheese, and ice-cream. The colloidal stability of casein micelles is described here by a calculation of the pair potential, containing the essential contributions of brush repulsion, electrostatic repulsion, and van der Waals attraction. The parameters required are taken from the literature. The results are expressed by the second osmotic virial coefficient and are quite consistent with experimental findings. It appears that the stability is mainly attributable to a steric layer of $\kappa$-casein, which can be described as a salted polyelectrolyte brush. (C) 2002 American Institute of Physics. [DOI: 10.1063/1.1484379]
\end{abstract}

\section{INTRODUCTION}

For millennia, man has known that milk flocculates and gels when it is acidified, as in yogurt production. The acidification is caused by lactic acid bacteria, which convert milk sugar into lactic acid. Near $p \mathrm{H} 4.8$ the onset of a macroscopic flocculation of the casein micelles in milk is observed. The colloidal stability of biological, i.e., waterborne, systems such as milk and blood is of great scientific interest and yet has hardly been described in a quantitative way. In order to understand the properties of casein micelles as present in milk it is necessary to appreciate their colloidal character. Nowadays the "hairy casein micelle" model of Holt ${ }^{1}$ is generally accepted and it helps the understanding of several dairy technological aspects of milk products. In this model the casein micelle is regarded as a colloidal particle, which is an associate of about a thousand small nanoclusters. These nanoclusters are the building block of the self-assembling casein micelle. ${ }^{1-3}$ The physiological function of casein micelles is to transport calcium phosphate to the neonate. ${ }^{4}$ To prevent calcination of the mammary gland, small calcium phosphate nuclei are covered by caseins, which are ampholytic proteins. The nanoclusters so formed $(20 \mathrm{~nm})^{5}$ associate further into casein micelles $(200 \mathrm{~nm})$; also see de Kruif and Holt. ${ }^{6}$ The casein micelle is further characterized by the presence of $\kappa$-caseins at the surface which protrude into the solvent. Actually, 63 of the 169 amino acids are on the outside of the casein micelles, while the other 106, which are more hydrophobic, are "inside" the casein micelle. ${ }^{6}$ The "exterior" part of the $\kappa$-caseins provides the steric stabilization of the casein micelles. In the "exterior" part there are 15 charged groups with an effective dissociation constant, $\mathrm{pK}_{\mathrm{a}}$, of 4.9. ${ }^{7}$ Casein micelles are polydisperse in size and can be characterized by a number-averaged radius of $100 \mathrm{~nm}^{8}$ Their size distribution is well described by a log-normal distribution, ${ }^{8}$ for instance giving a weight-averaged radius of

\footnotetext{
a) Author to whom correspondence should be addressed: Electronic mail: dekruif@nizo.nl
}

$140 \mathrm{~nm}$, which is in agreement with light-scattering experiments by Bauer et al. ${ }^{9}$ Further, at neutral $p \mathrm{H}$ casein micelles behave effectively as hard spheres, as can be derived from the concentration dependence of their self- and collective diffusion ${ }^{10}$ and their rheological behavior. ${ }^{11}$ When skim milk is acidified the transport properties become those of adhesive hard spheres. ${ }^{10,12}$ This means that the interactions between casein micelles change from hard-sphere-like to effectively attractive during a $p \mathrm{H}$ decrease. In cheese-making the steric stabilization (the "hairy" layer) is removed enzymically and that induces gelation into the cheese curd. ${ }^{6}$ Here we show that the stability of casein micelles in milk upon acidification and renneting can be described quantitatively using current knowledge of the pair potential. Our approach may also serve as an example for a quantitative understanding of the stability of complex colloidal dispersions in aqueous solutions.

In the second half of the last century the understanding of the stability of colloidal dispersions increased enormously. Of paramount importance was the development of the Derjaguin-Landau-Verwey-Overbeek (DLVO) theory (see Refs. 13 and 14 or an overview in Ref. 15). Payens, ${ }^{16,17}$ and Walstra and Jenness, ${ }^{4}$ discussed the application of these theories to describe the colloidal stability of a dispersion of casein micelles in milk. From their work it follows that the electrostatic repulsion is not strong enough to compensate the van der Waals attraction and prevent flocculation. So, according to the DLVO theory casein micelles are not stable when present in milk. Paradoxically, they are in fact stable and adding kitchen salt does not lead to flocculation. During the 1970s it was realized that steric stabilization of colloids was present in many systems, ${ }^{18}$ and Holt ${ }^{19}$ noticed that this was also relevant for the description of the interaction between casein micelles. Subsequently, in the last decade it became evident that $\kappa$-casein can be regarded as a brush of a grafted polymer at the surface of the casein micelles. Strictly speaking, the $\kappa$-caseins have to be regarded as block copolymers, with a block absorbed in the micelle and a nonadsorbing block sticking into the solution. De Kruif and Zhulina ${ }^{20}$ modeled $\kappa$-casein as a polyelectrolyte brush, and their calcu- 
lations explain many features of the stability of casein micelles during renneting. From the foregoing it follows that the knowledge of and insights into casein micelles and their interactions have increased significantly in recent years. Therefore it is intriguing to try to answer the question raised by Holt and Horne: "Can we quantify the strengths of these micellar interactions?" Here we calculate the pair potential between casein micelles and subsequently the second osmotic virial coefficient, $\mathrm{B}_{2}$. We calculate $\mathrm{B}_{2}$ since this parameter can be used to calculate both equilibrium and transport properties, and is an excellent parameter to characterize the colloidal stability.

\section{Theoretical section}

The interaction potential contains many contributions, of which only a few are probably relevant. The three relevant types of interaction that play the most important roles in the stability of casein micelles are van der Waals attraction, electrostatic repulsion, and polymer brush repulsion. Van der Waals dispersion forces arise through mutually fluctuationinduced polarization of the electrons in atoms. For the van der Waals attraction, $W_{\mathrm{vdW}}(h)$, between two spheres separated by a distance $h$, where $h=r-2 a, a$ is the sphere radius and $r$ is the sphere center to center distance, one finds ${ }^{21}$

$$
\begin{aligned}
\frac{W_{\mathrm{VdW}}(h)}{k T}= & -\frac{A}{6}\left(\frac{2 a^{2}}{(h+2 a)^{2}-4 a^{2}}+\frac{2 a^{2}}{(h+2 a)^{2}}\right. \\
& \left.+\ln \left\{\frac{(h+2 a)^{2}-4 a^{2}}{(h+2 a)^{2}}\right\}\right),
\end{aligned}
$$

where $A$ is the Hamaker constant. We may use results of the Hamaker constant as found for proteins, which are of the order 1-3 kT. ${ }^{22,23}$ Recently, Schaink and Smit ${ }^{24}$ calculated the Hamaker constant of $\beta$-lactoglobulin as $\sim 5 \mathrm{kT}$ near the iso-electric point. Gripon et al. ${ }^{25}$ did scattering experiments on lysozyme solutions from which they derived a Hamaker constant of $\sim 7 \mathrm{kT}$ at $25^{\circ} \mathrm{C}$. The protein density of casein micelles is about 6 times less than that of $\beta$-lactoglobulin and lysozyme and therefore we estimated $A$ as $1.0 \mathrm{kT}$. The gel strengths of $\beta$-lactoglobulin and lysozyme gels are much greater than that of a casein micelle gel, which is consistent with the much smaller Hamaker constant of casein micelles.

At the physiological and near-neutral $p \mathrm{H}$ of 6.7 as in milk the caseins carry charges from dissociated acid and basic protein groups. Charge distributions in solution are usually described by a Boltzmann distribution. The range of electrostatic interaction is expressed by the Debye length $\kappa^{-1}$ :

$$
\kappa^{-1}=\sqrt{\frac{\varepsilon_{0} \varepsilon_{r} R T}{F^{2} I}},
$$

where $\varepsilon_{0}$ is the permittivity in vacuum, $\varepsilon_{r}$ is the relative permittivity, $F$ is Faraday's constant, and $I$ is the ionic strength, which is $0.08 \mathrm{M}$ in milk, ${ }^{4}$ corresponding to a Debye length of the order of $1 \mathrm{~nm}$. The electrostatic repulsion between casein micelles in milk is therefore short-ranged. The expression for the interaction potential between two spheres due to electrostatic repulsion $W_{\text {er }}(h)$ can be derived from a linear approximation to the Boltzmann charge distribution around two colloids: ${ }^{26}$

$$
\frac{W_{\mathrm{er}}(h)}{k T}=-2 \pi a \varepsilon_{0} \varepsilon_{r} \Psi^{2} \ln (1+\exp (\kappa h)),
$$

where $\Psi$ is the surface potential. The surface potential cannot be measured directly but the zeta potential, which approximates the potential at a certain distance from the particle surface, can be measured electrokinetically, and was found to be close to $-8 \mathrm{mV}$ at neutral $p \mathrm{H}^{27}$ On decreasing the $p \mathrm{H}$, Schmidt and Poll measured a zeta-potential that approached a value of $0 \mathrm{mV}^{26}$ When the casein micelles become unstable, it is hard to measure the zeta-potential properly, but, considering the point of zero charge (PZC) values of the caseins in the micelles, it is expected that the zetapotential will be zero around $p \mathrm{H} 4.8$ and that it will become positive at lower $p \mathrm{H}$ values.

To calculate the steric (repulsive) interaction due to brush repulsion we will first evaluate the relation between the brush height and the $p \mathrm{H}$ for $\kappa$-caseins in skim milk and will follow the theory of grafted weak polyacids proposed by Israels et $a .^{28}$ As discussed by De Kruif and Zhulina, ${ }^{20}$ $\kappa$-caseins can be described as charged brushes in the ("salted brush") regime. In this regime the salt concentration is such that it penetrates the brush and screens the electrostatic interactions between the charged polyacid groups; $\kappa^{-1} \ll H$, where $H$ is the brush height. Then the polyacid brush is quasi-neutral; its characteristics are identical to those of neutral brushes. Consequently, in the strong stretching approximation, the brush height then reads ${ }^{28,29}$ as

$$
H=N b\left(\frac{8 \nu^{\mathrm{eff}} \theta}{\pi^{2}}\right)^{1 / 3},
$$

where $N$ is the number of segments of the brush, each having a length $b$. The quantity $\theta$ is the grafting density: the fraction of "sites" occupied by the brushes at the surface. The parameter $\sigma\left(\mathrm{m}^{-2}\right)$ is the grafting density $\theta$ divided by the surface area occupied by a brush. The effective excluded volume is denoted by $\nu^{\text {eff }}$ and is defined as

$$
\nu^{\mathrm{eff}}=\nu+\frac{\alpha^{2}}{\phi_{s}},
$$

where $\nu$ is the (dimensionless) excluded volume per segment (normalized with the segment volume), $1-2 \chi$ (with $\chi$ the Flory-Huggins interaction parameter), and $\phi_{s}$ is the salt volume fraction. We take a very simple model for the dissociation of the polyacid brush in which we assume that the various dissociating groups do not affect one another, which leads to the following relation between the $p \mathrm{H}$ and the degree of dissociation $\alpha$ :

$$
\alpha=\frac{K_{a}}{K_{a}+\left[\mathrm{H}^{+}\right]},
$$

where $\left[\mathrm{H}^{+}\right]$is the proton concentration $\left(\equiv 10^{-p \mathrm{H}}\right)$.

The above offers us a simplified model for the brush height as a function of $p \mathrm{H}$. It is realized that Eq. (4) loses its 
applicability at low $p \mathrm{H}$ since then it is less stretched. It is known that a $\kappa$-casein brush collapses at low $p \mathrm{H}^{20}$ Therefore for $\chi$ 's close to $\frac{1}{2} \mathrm{Eq}$. (4) still yields a proper qualitative description of the brush height at low $p \mathrm{H}$; it predicts the collapse. For the interaction potential between hard parallel plates at a distance $h$ carrying brushes we use the Alexander-de Gennes theory, ${ }^{30,31}$ which leads to the following expression for the force between two parallel flat plates:

$$
\begin{aligned}
\frac{K_{\text {brush }}(h)}{k T} & =\sigma^{3 / 2}\left[\left(\frac{2 H}{h}\right)^{9 / 4}-\left(\frac{h}{2 H}\right)^{3 / 4}\right], \quad \text { for } h<2 H, \\
& =0, \quad \text { for } h>2 H,
\end{aligned}
$$

where the first term between the square brackets represents the increase in the osmotic pressure (leading to repulsion) due to the increase of the brush concentration between the plates and the second term represents the change in elastic free energy of the brush upon compression. The interaction potential between two flat surfaces can be obtained by integrating $K_{\text {brush }}(h)$ over the plate separation distance. By subsequently applying the Derjaguin approximation ${ }^{32}$ to the potential between two flat surfaces, the following expression for the interaction potential between two spheres, with radius $a$, is obtained: ${ }^{33,34}$

$$
\begin{aligned}
\frac{W_{\text {brush }}(h)}{\mathrm{kT}}= & \infty \quad \text { for } h<0 \\
= & \frac{16 \pi a H^{2} \sigma^{3 / 2}}{35}\left[\left(28\left(\frac{2 H}{h}\right)^{1 / 4}-1\right)\right. \\
& \left.+\frac{20}{11}\left(1-\left(\frac{h}{2 H}\right)^{11 / 4}\right)+12\left(\frac{h}{2 H}-1\right)\right], \\
& \text { for } 0<h<2 H \\
= & 0 \text { for } h>2 H .
\end{aligned}
$$

The Derjaguin approximation is quite reasonable as long as the range of the interaction potential is much smaller than the sphere radius, which is the case for casein micelles in milk. In our model calculations we describe the brush part of the $\kappa$-casein as a salted brush containing 15 weakly charged groups that have a $p K_{a}$ of 4.9. The part of the $\kappa$-casein that protrudes into the solvent contains 63 amino acids, each having a length of approximately $0.32( \pm 0.04) \mathrm{nm}$ (calculated using the computer program CHARMM). The segment length used in our model [b in Eq. (4)] should thus be at least 0.32 $\mathrm{nm}$. The grafting density $\sigma$ can be estimated by a calculation of the surface occupied by the $\kappa$-caseins at the casein micelle surface. The total amount of surface of casein micelles $A_{\mathrm{CM}}$ in the system per unit volume equals

$$
A_{\mathrm{CM}}=6 \phi \frac{\int_{0}^{\infty} \Psi(D) D^{2} d D}{\int_{0}^{\infty} \Psi(D) D^{3} d D},
$$

where $\Psi(D)$ is the size distribution of the casein micelles, each having a diameter $D$. De Kruif ${ }^{8}$ analyzed this size distribution and found that it can be described by a log-normal distribution. In Eq. (9), $\phi$ is the volume fraction of casein

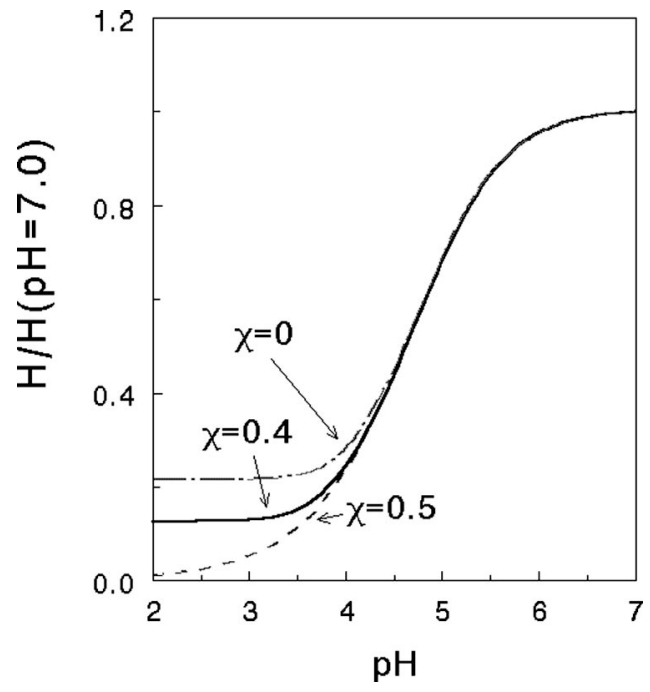

FIG. 1. Relative root-mean-square brush height of $\kappa$-caseins at the surface of casein micelles as a function of $p \mathrm{H}$ for two solvent qualities for the brush, $\chi=0$, and 0.4, as calculated from Eqs. (4)-(6).

micelles, which equals 0.13 in unheated skim milk. ${ }^{11}$ This yields a total surface per volume $A_{\mathrm{CM}}$ of $1.76 \times 10^{3} \mathrm{~m}^{2} / \mathrm{L}$ skim milk. In milk one has $3.3 \mathrm{~g} \kappa$-casein/L, which corresponds to a brush density of $1.88 \mathrm{mg} / \mathrm{m}^{2}$. The molar mass of $\kappa$-casein is $19032 \mathrm{~g} / \mathrm{mol}$, the brush density thus corresponds to approximately 7500 chains per casein micelle, and the average distance between the chains is $4 \mathrm{~nm}$. The area occupied by one chain at the surface is at least the squared length of an amino acid group in the $\kappa$-casein brush, where the length equals about $0.32 \mathrm{~nm}$. We therefore estimate $\theta$ as 0.006 . The salt volume fraction is estimated as 0.01 (salt concentration $\approx 7.7$ times salt volume fraction ${ }^{28}$ ). We set the segment length at $0.60 \mathrm{~nm}$ in order to attain a brush height of $7.0 \mathrm{~nm}$, which is in fair agreement with dynamic lightscattering experiments. ${ }^{10}$

\section{RESULTS AND DISCUSSION}

In Fig. 1 we plot the relative brush height $H / H(p \mathrm{H} 7)$, where $H(p \mathrm{H} 7)$ is the brush height at $p \mathrm{H} 7.0$ for three solvent qualities of the brush; $\chi=0,0.4$, and 0.5 . The brush height $H$ is calculated from Eqs. (4)-(6) using the parameters given above. We observe that the relative brush height starts to decrease gradually below $p \mathrm{H} \mathrm{6}$, and decreases very rapidly with decreasing $p \mathrm{H}$ below $p \mathrm{H} \mathrm{5.3.} \mathrm{The} \mathrm{curves} \mathrm{are}$

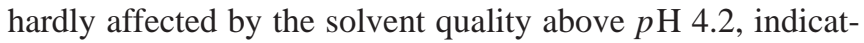
ing that the decrease in relative brush height is not sensitive to the solvent quality in the relevant $p \mathrm{H}$ range where flocculation occurs. In the rest of this paper we use the results for $\chi=0.5$, since that correlates with a collapse of the brush at low $p \mathrm{H}$. We can now use the $p \mathrm{H}$-brush height dependence to calculate its effect on the interaction between the casein micelles using the Alexander-de Gennes approximation.

In Figure 2 we plot the various contributions to the total interaction potential. Plotted are the van der Waals attraction [Eq. (1) using $a=100 \mathrm{~nm}$ as the radius of a casein micelle ${ }^{8}$, electrostatic repulsion [using Eq. (2) with $+20 \mathrm{mV}$ as the surface potential at $p \mathrm{H} 6.7$ which can be estimated from the 


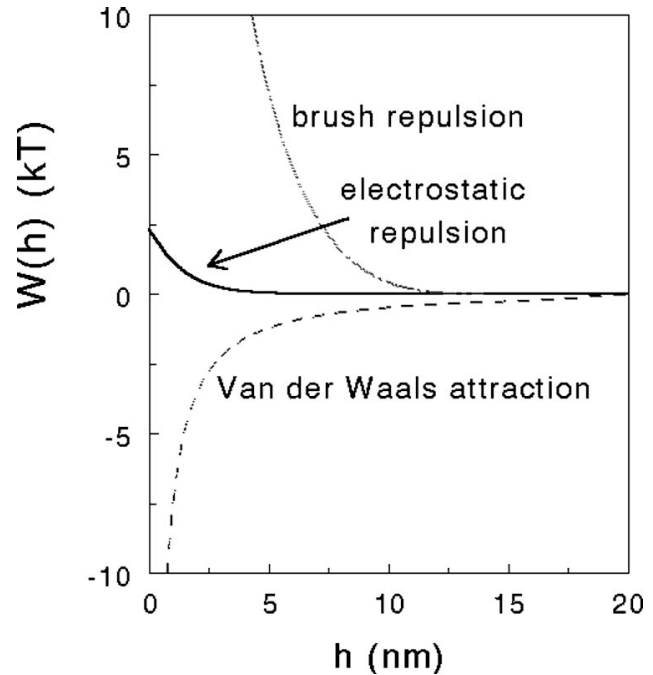

FIG. 2. Contributions to the interaction potential between casein micelles at $p \mathrm{H} 6.7$ due to brush and repulsion, electrostatics, and van der Waals attraction.

zeta-potential measured by Schmidt and $\left.\mathrm{Poll}^{27}\right]$, and brush repulsion [Eq. (8)] at $p \mathrm{H} \mathrm{6.7.} \mathrm{On} \mathrm{lowering} \mathrm{the} p \mathrm{H}$ it approaches the PZC and one even expects a smaller contribution of the electrostatic repulsion. Close to the flocculation $p \mathrm{H}$ (4.8) hardly any net charge remains. We approximated the surface potential $\psi$ to: $\psi \sim(\mathrm{PZC}-p \mathrm{H})$ to take this effect into account. This gives a reasonable description of the zetapotential measurements of Schmidt and Poll. From the potentials plotted in Fig. 2 it becomes clear that we can neglect electrostatic repulsion since brush repulsion overwhelms it. We note that inserting electrostatic repulsion hardly affects the results.

The total interaction potential is now taken as the sum of Eqs. (1) and (8), and is plotted for a few $p \mathrm{H}$ values in Fig. 3. The attraction becomes significant on lowering the $p \mathrm{H}$ below 5. This is due to the significant reduction of the brush height. Below $p \mathrm{H} 5$ the brush repulsion becomes significantly weaker, while the casein micelles will probably become slightly positively charged. In this $p \mathrm{H}$ the repulsion is overwhelmed by the van der Waals attraction, which leads to a strong net attraction. Given the total interaction potential, the equilibrium (and transport) properties of the system can be evaluated using statistical mechanics. ${ }^{35}$ From statistical mechanics we can calculate the second osmotic virial coefficient $B_{2}$ from $W_{\text {tot }}(r)$, with $r=h+2 a$ :

$$
B_{2}=\frac{2 \pi}{V_{c}} \int_{0}^{\infty} r^{2}\left[1-\exp \left(-\frac{W_{\text {tot }}(r)}{k T}\right)\right] d r
$$

where $W_{\text {tot }}(h)$ is the total interaction potential between the casein micelles. For any interaction potential this then yields $B_{2}$, which becomes negative if the net attraction is sufficiently strong. In the calculations $h_{0}=0.5 \mathrm{~nm}$ was taken as a cut-off length, that value of $h$ below which $W_{\text {tot }}(h)$ is defined as $\infty$, since it would be impossible to compress the spheres to $h=0$. A reasonable estimate for the value of $B_{2}$ where colloidal suspensions become unstable is $B_{2} \approx-6^{36}$. In Fig. 4 the second osmotic virial coefficient is given as a function of

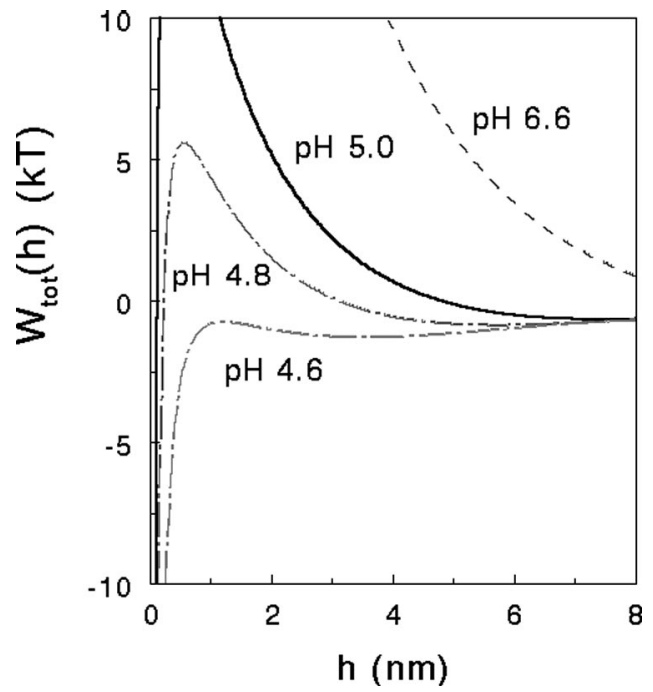

FIG. 3. Calculated total interaction potential between casein micelles for various $p \mathrm{H}$ values, as indicated.

the $p \mathrm{H}$. At $p \mathrm{H} 7 B_{2}$ has a value of 4.4 , which is very close to 4 , the value for hard spheres. ${ }^{35}$ Upon decreasing the $p \mathrm{H}$ $B_{2}$ decreases very gradually up to $p \mathrm{H} 4.6$, after which it drops very rapidly. It is clear that the instability region is reached quite suddenly, and at $p \mathrm{H} 4.5$ one expects the casein micelles to become unstable, as estimated from the value where $B_{2}$ becomes -6 . This $p \mathrm{H}$ value of 4.5 is close to the onset of flocculation, which is usually referred to as $p \mathrm{H}$ 4.7-4.8. ${ }^{4}$ It is also known that decreasing the amount of $\kappa$-casein brushes, as occurs during renneting, ${ }^{4,37}$ the first stage in the cheese-making process, increases the flocculation $p \mathrm{H}^{8,10}$ In Fig. 4 we therefore also added the results for the situation that $20 \%, 50 \%$, and $60 \%$ of $\kappa$-casein at the casein micelle surface is cleaved off. It is clear that the sudden decrease of the second osmotic virial coefficient now takes place at a higher $p \mathrm{H}$ than 4.9: 4.7 for $20 \% \kappa$-casein cleavage, 5.1 for $50 \%$ cleavage, and 5.7 for $60 \%$ of cleavage. This effect thus corresponds to experimental findings. ${ }^{8,10}$

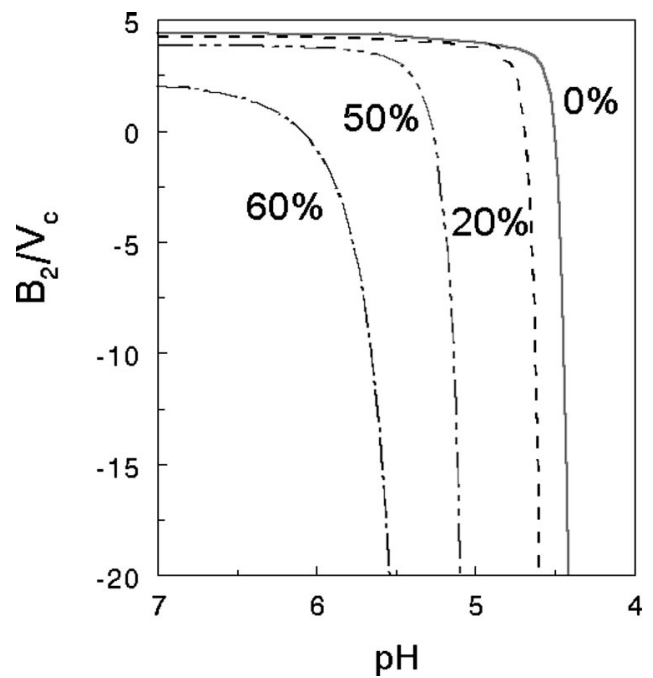

FIG. 4. Second osmotic virial coefficient of casein micelles as calculated from Eqs. (10) and (11) as a function of the $p \mathrm{H}$ (full curve). The dashed curves refer to the calculated values of $B_{2}$ for casein micelles of which a certain fraction of brushes is cleaved off. 


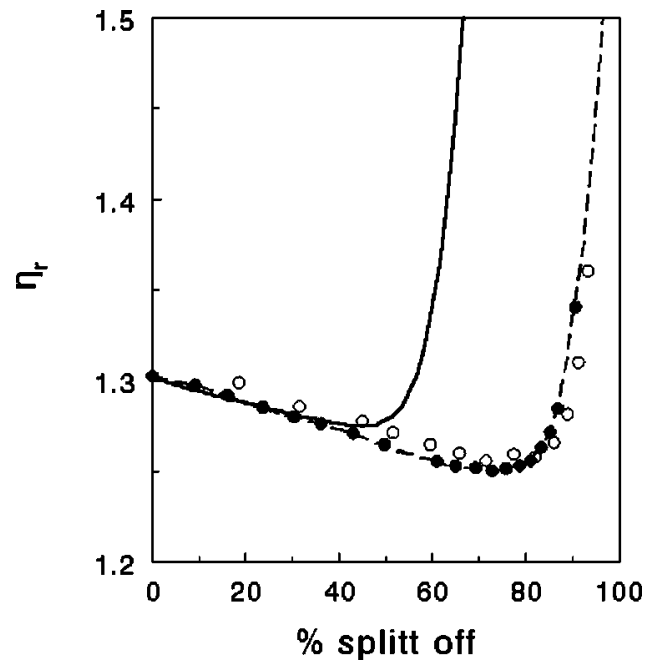

FIG. 5. Viscosity of a skim milk dispersion as a function of the percentage of $\kappa$-casein cleaved off during renneting. The experimental data are given as the data points from Ref. 40 and the full curve is calculated using the model proposed here. The dashed curve represents the prediction if the cleaved-off concentrations where the divergence of the viscosity is predicted matches.

Since the potential is short-ranged it is justifiable to refer to the adhesive hard sphere theory of Baxter, ${ }^{38}$ which is often used to describe equilibrium and transport properties. The effective attraction in this model is expressed by the Baxter parameter $\tau_{\mathrm{B}}$, which relates to $B_{2}=4-1 / \tau_{\mathrm{B}}$. For the relative viscosity Cichocki and Felderhof ${ }^{39}$ derived the following equation for adhesive hard spheres:

$$
\eta_{r}=1+\frac{5}{2} \phi+\left(5.9+\frac{1.9}{\tau_{\mathrm{B}}}\right) \phi^{2} .
$$

In Fig. 5 we have plotted the experimental data of De Kruif et al. ${ }^{40}$ who measured the relative viscosity of a skim milk dispersion with a volume fraction of 0.11 during renneting. The rennet time was translated to the percentage of $\kappa$-casein cleaved off by using their data. Our data are given in this plot as well. Both experimental data and our theoretical prediction exhibit identical behavior. After the decrease of relative viscosity (due to a decrease of the radii of the micelles and thus of the effective volume fraction) there is a sudden sharp upturn leading to a strong increase in the relative viscosity. There is, however, a shift in the percentage of cleavage where the upturn appears. Whereas our theory predicts that this happens at $60 \%$, experimental data show that this point lies around $80 \%$. In our analysis we assumed that all $\kappa$-casein can be cut off and also that there are no other brushes present. There is evidence ${ }^{6}$ that there is also a small fraction of $\beta$-casein brushes that are not cut off. They may enhance the stability, which may explain the difference we find.

\section{CONCLUSION}

The generally accepted and used models presented adequately describe the behavior and properties of casein micelles as found in milk. Using parameters derived from independent experimental data we were able to describe the stability of casein micelles during the initial stages of the yogurt and cheese-making processes without using additional adjustable parameters. The results show that the steric stabilization generated by a relatively sparse brush is the most important stabilizing factor. Although our model is quite simple and contains several assumptions, inevitable for such a complex biological association colloid as a casein micelle, it gives a good description of the stability of casein micelles on lowering the $p \mathrm{H}$ and this understanding should be helpful for dairy technological developments.

\section{ACKNOWLEDGMENTS}

We thank C. Holt, Hannah Research Institute, Ayr, Scotland and W. R. Russel, Department of Chemical Engineering, Princeton University, USA, for useful discussions.

${ }^{1}$ C. Holt, in Advances in Protein Chemistry, edited by C. B. Anfinsen, J. D. Edsall, F. R. Richards, and D. S. Eisenberg (Academic, San Diego, 1992), Vol. 43, pp. 63-151

${ }^{2}$ C. Holt and D. S. Horne, Neth. Milk Dairy J. 50, 85 (1996).

${ }^{3}$ D. S. Horne, Int. Dairy J. 8, 171 (1998).

${ }^{4}$ P. Walstra and R. Jenness, Dairy Chemistry and Physics (Wiley, New York, 1984).

${ }^{5}$ C. Holt, P. A. Timmins, N. Errington, and J. Leaver, Eur. J. Biochem. 252, 73 (1998).

${ }^{6}$ C. G. De Kruif and C. Holt, "Casein micelle structure, functions and interactions," to be published as Chap. 3 in 2001 in Advances in Dairy Chemistry, edited by P. F. Fox and P. L. H. McSweeney (in press).

${ }^{7}$ H. E. Swainsgood, "Proteins," in Advances in Dairy Chemistry, edited by P. F. Fox (Elsevier Applied Science, London, 1992), Vol. 1.

${ }^{8}$ C. G. De Kruif, J. Dairy Sci. 81, 3019 (1998).

${ }^{9}$ R. Bauer, M. Hansen, L. Ogendal, S. B. Lomholt, and K. B. Qvist, J. Chem. Phys. 103, 2725 (1995).

${ }^{10}$ C. G. De Kruif, Langmuir 8, 2932 (1992).

${ }^{11}$ Th. J. M. Jeurnink and C. G. De Kruif, J. Dairy Res. 60, 139 (1993).

${ }^{12}$ C. G. De Kruif, J. Colloid Interface Sci. 185, 19 (1997).

${ }^{13}$ B. V. Derjaguin and L. D. Landau, Acta Physicochim. URSS 14, 633 (1941).

${ }^{14}$ E. J. W. Verwey and J. Th. G. Overbeek, Theory of the Stability of Lyophobic Colloids (Elsevier, Amsterdam, 1948).

${ }^{15}$ J. Th. G. Overbeek, in Colloidal Dispersions, edited by J. W. Goodwin (Royal Society of Chemistry, London, 1981).

${ }^{16}$ T. A. J. Payens, J. Dairy Sci. 49, 1317 (1966).

${ }^{17}$ T. A. J. Payens, J. Dairy Res. 46, 291 (1979).

${ }^{18}$ D. H. Napper, Trans. Faraday Soc. 64, 701 (1968).

${ }^{19}$ C. Holt, "The stability of bovine casein micelles," in Proceedings of the International Conference on Colloid and Interface Science, Vol. 1, Akadémiai Kiadó, edited by E. Wolfram, 1975, pp. 641-644.

${ }^{20}$ C. G. De Kruif and E. B. Zhulina, Colloids Surf., A 117, 151 (1996).

${ }^{21}$ H. C. Hamaker, Physica (Amsterdam) 4, 1058 (1937).

${ }^{22}$ J. Visser, Adv. Colloid Interface Sci. 3, 331 (1972).

${ }^{23}$ S. Nir, Prog. Surf. Sci. 8, 1 (1977).

${ }^{24}$ H. M. Schaink and J. A. M. Smit, Phys. Chem. Chem. Phys. 2, 1537 (2000).

${ }^{25}$ C. Gripon, L. Legrand, I. Rosenman, O. Vidal, M. C. Robert, and F. Boué, J. Cryst. Growth 178, 575 (1997).

${ }^{26}$ R. Hogg, T. W. Healy, and D. W. Fuerstenau, Trans. Faraday Soc. 62, 1638 (1966).

${ }^{27}$ D. G. Schmidt and J. K. Poll, Neth. Milk Dairy J. 40, 269 (1986).

${ }^{28}$ R. Israels, F. A. M. Leermakers, and G. J. Fleer, Macromolecules 27, 3087 (1994).

${ }^{29}$ C. M. Wijmans, J. M. H. M. Scheutjens, and E. B. Zhulina, Macromolecules 25, 2567 (1992).

${ }^{30}$ S. Alexander, J. Phys. (France) 38, 983 (1977).

${ }^{31}$ P. G. de Gennes, Adv. Colloid Interface Sci. 27, 189 (1987)

${ }^{32} \mathrm{~J}$. Lyklema, Fundamentals of Interface and Colloid Science (Academic, London, 1991), Vol. 1.

${ }^{33}$ G. J. C. Braithwaite, P. F. Luckham, and A. M. Howe, J. Colloid Interface Sci. 213, 525 (1999). 
${ }^{34}$ C. N. Likos, K. A. Vaynberg, H. Löwen, and N. J. Wagner, Langmuir 16, $4100(2000)$

${ }^{35}$ D. A. McQuarrie, Statistical Mechanics (Harper \& Row, New York, 1976).

${ }^{36}$ G. A. Vliegenthart and H. N. W. Lekkerkerker, J. Chem. Phys. 112, 5364 (2000).
${ }^{37}$ D. A. Pink, B. Quinn, and K. Baskin, Langmuir 10, 2559 (1994).

${ }^{38}$ R. J. Baxter, J. Chem. Phys. 49, 2770 (1968).

${ }^{39}$ B. Cichocki and B. U. Felderhof, J. Chem. Phys. 89, 3705 (1988).

${ }^{40}$ C. G. De Kruif, Th. J. M. Jeurnink, and P. Zoon, Neth. Milk Dairy J. 46, 123 (1992). 